УДК 631.373

\author{
А.Ю. Ткаченко ${ }^{1,2}$, И.А. Лобач ${ }^{1,3}$ \\ ${ }^{1}$ Институт автоматики и электрометрии Сибирского отделения РАН, \\ Новосибирск, Россия \\ ${ }^{2}$ Новосибирский государственный технический университет, \\ Новосибирск, Россия \\ 3 Лаборатория фротоники Пермского научного центра \\ Уральского отделения РАН, Пермь, Россия
}

УСТРОЙСТВО ОПРОСА ВОЛОКОННЫХ СЕНСОРОВ НА БАЗЕ ВОЛОКОННОГО ЛАЗЕРА
С САМОСКАНИРОВАНИЕМ ЧАСТОТЫ

Разработан полностью волоконный одночастотный иттербиевый лазер с самосканированием частоты в области 1058-1076 нм и выходной мощностью более 50 мВт с возможностью управления областью сканирования с помощью температуры активного волокна. На основе лазера реализован прототип устройства опроса волоконных сенсоров, который был успешно апробирован на линии, состоящей из 6 ВБР-датчиков. Точность определения длины волны отражения датчиков оценена как 2 пм. Рассмотрены различные варианты улучшения характеристик устройства.

Ключевые слова: волоконный лазер, устройство опроса, брэгговская решетка, сканирование частоты.

\author{
A.Iu. Tkachenko ${ }^{1,2}$, I.A. Lobach ${ }^{1,3}$ \\ ${ }^{1}$ Institute of Automation and Electrometry of the Siberian Branch of the Russian \\ Academy of Sciences, Novosibirsk, Russian Federation \\ ${ }^{2}$ Novosibirsk State Technical University, Novosibirsk, Russian Federation \\ ${ }^{3}$ Laboratory of Photonics, Perm Scientific Center of the Ural Branch of the Russian \\ Academy of Sciences, Perm, Russian Federation

\section{FIBER SENSOR INTERROGATOR BASED ON SELF-SWEEPING FIBER LASER}

In this paper single-frequency ytterbium self-sweeping all-fiber laser with sweeping range of 1058-1076 nm and output power of more than $50 \mathrm{~mW}$ with temperature-controlled sweeping range is developed. A prototype of fiber sensor interrogator based on the laser is designed, which has been successfully tested on the sensor line consisting of 6 FBG sensors. The accuracy of measured wavelength of FBG reflection is estimated as $2 \mathrm{pm}$. The possible improvements of the interrogator are discussed.

Keywords: fiber laser, an interrogator, Bragg grating, frequency sweeping. 


\section{Введение}

С развитием технологий в промышленности, строительстве, нефте- и газодобыче, а также других сферах деятельности человека повышается и сложность конструкций зданий, сооружений, различных технических агрегатов и устройств. Как следствие, особо важную роль играют диагностика и мониторинг их текущего технического состояния. Одними из самых современных технологий контроля температуры, a также геометрических параметров объектов являются волоконные сенсорные системы [1]. В качестве датчиков в волоконных сенсорных системах применяются волоконные брэгговские решетки (ВБР), которые представляет собой периодическую модуляцию показателя преломления сердцевины оптоволокна [2]. Такая структура способна отражать излучение в определенном спектральном диапазоне. Длина волны отраженного излучения зависит от среднего показателя преломления и периода структуры. В свою очередь эти величины зависят от внешних параметров, а именно - от температуры и механического напряжения [2]. Таким образом, вычисляя разницу между отраженными длинами волн до и после воздействия, можно определить величину воздействия (например, изменение температуры). Поскольку датчики являются оптическими, они могут применяться в условиях повышенной взрывоопасности, сильных электромагнитных и радиочастотных воздействий, в контакте с горючими смесями. На основе ВБР-датчиков можно создавать квазираспределенную сенсорную линию путем записи в одном оптоволокне большого количества ВБР, каждая из которых дает отклик на собственной длине волны.

Для измерения отклика сенсорной линии применяется устройство опроса, которое представляет собой не что иное, как спектроанализатор. В существующих вариантах реализации устройства опроса применяется либо широкополосный источник в паре с решеточным анализатором спектров, либо перестраиваемый лазер в паре с фотодетекторами [3]. В коммерчески доступных устройствах опроса вариант с решеточными анализаторами спектров не используется по причине дороговизны таких приборов. На сегодняшний день существует множество исследований, посвященных разработке перестраиваемых волоконных лазерных источников с целью создания устройств опроса волоконных сенсорных систем, но стоит отметить, что практически во всех этих работах применяются перестраиваемые спектральные эле- 
менты, которые управляются либо вручную, либо внешней электроникой. Для перестройки используются интерферометры Фабри-Перо [4], дифракционные решетки [5] или призмы, перестраиваемые ВБР [6]. Также известны работы, в которых перестройка частоты осуществляется за счет модуляции параметров лазера (например, тока в [7]). Кроме того, существует множество работ, посвященных созданию волоконного лазера с перестройкой частоты на основе синхронизации мод в Фурье-пространстве [8]. Во всех этих случаях также необходим управляющий драйвер. Стоит отметить, что управляющий драйвер совместно с перестраиваемым спектральным фильтром являются достаточно дорогостоящими элементами, что приводит к удорожанию как перестраиваемого лазера, так и всего устройства опроса сенсорной системы. С другой стороны, есть работы [9-19], в которых показано, что перестройка лазерной частоты может происходить без управляющего драйвера. Например, подобная перестройка частоты происходит в лазерах с самоиндуцированным сканированием (самосканированием) частоты.

Эффект самосканирования частоты генерации в волоконных лазерах был открыт в рубиновом лазере спустя несколько лет после создания первого лазера [9]. В волоконных же лазерах эффект был обнаружен всего несколько лет назад $[10,11]$. Самосканирование связано с формированием динамических решеток коэффициента усиления и показателя преломления из-за эффекта выжигания дыр стоячей волной в линейном резонаторе [12]. Долгоживущие решетки, индуцированные генерируемыми продольными модами, влияют на выход в генерацию новых мод, что приводит к динамике спектра генерации. На сегодняшний день получены различные варианты проявления эффекта. Например, длина волны генерации может как увеличиваться во времени, так и уменьшаться, периодически возвращаясь в начальное состояние. В каждый момент времени спектр генерации может быть как многочастотным, так и одночастотным (т.е. состоящим из одной продольной моды), а диапазон сканирования - более 20 нм [12]. Несмотря на то что основная доля работ посвящена изучению эффекта в иттербиевом волоконном лазере, генерирующем в области 1 мкм, предпринимаются попытки расширения диапазона лазера с помощью других активных сред. На сегодняшний день эффект самосканирования продемонстрирован в висмутовом лазере с диапазоном сканирования 10 нм в области 
1,46 мкм [13], в эрбиевым лазере с диапазоном сканирования менее 1 нм в области 1,5 мкм [14], в тулий-гольмиевом волоконном лазере с диапазоном сканирования 17 нм в области 1,9 мкм [15]. Также есть работа [16], где подобный лазер был применен для высокоразрешающей характеризации узких спектральных структур ВБР с фазовым сдвигом. Последняя работа показывает, что волоконный лазер с самосканированием частоты может конкурировать с другими перестраиваемыми источниками в ряде приложений.

Цель данного исследования заключается в разработке устройства опроса ВБР-сенсоров на основе волоконного лазера с самосканированием частоты и его апробации.

\section{Эксперимент}

\section{1. Волоконный лазер с пассивным сканированием частоты}

Для устройства опроса был реализован иттербиевый волоконный лазер с накачкой в оболочку, работающий в режиме самосканирования частоты (рис. 1, a). Схема лазера аналогична схеме, представленной в работе [11], но отличается тем, что все компоненты были с сохранением линейной поляризации. Как было показано в работе [12], использование поляризационных элементов позволяет существенно улучшить стабильность лазерной генерации. Резонатор лазера был образован широкополосным и плотным волоконным зеркалом и прямым сколом волокна в качестве выходного зеркала. Также в резонатор был вставлен волоконный ответвитель 50/50, аналогично работе [11]. Таким образом, эффективный коэффициент отражения составил порядка $1 \%$, но при этом половина выходящей мощности излучения была сосредоточена в волокне и может быть использована в волоконно-оптических схемах. Для исключения обратного влияния на генерацию на выход лазера был поставлен изолятор. В качестве активной среды было выбрано иттербиевое волокно с двойной оболочкой и сохранением поляризации (PM-YDF-5/130Nufern) длиной 3 м. Длина активной среды была подобрана таким образом, чтобы обеспечить максимальный диапазон сканирования [17], который составлял более 18 нм (рис. 1, б). Активная среда накачивалась многомодовым лазерным диодом мощностью до 9 Вт через объединитель накачки. Также в схеме особое внимание было уделено динамике интенсивности, которая, как было показано в ра- 
боте [12], в первую очередь определяется положением активного световода в резонаторе. Подобрав длину пассивного волокна, находящегося между объединителем накачки и плотным зеркалом (см. рис. $1, a$ ), $\sim 10$ м, получили режим одночастотного самосканирования частоты $[12,16-18]$. В этом случае динамика интенсивности состоит из периодических микросекундных импульсов, лазерная частота меняется между импульсами на одну частоту межмодовых биений ( 5,5 МГц), а спектральная ширина каждого импульса составляет порядка 1 МГц. Для управления спектральным диапазоном сканирования в лазере была реализована возможность изменения температуры активного волокна $T_{1}$ и лазерного диода накачки $T_{2}$ [17]. Для этого активное волокно было намотано на термостабилизированную катушку с элементом Пельтье, которая позволяет нагревать световод от 10 до $50{ }^{\circ} \mathrm{C}$, что приводит к смещению области сканирования на 8 нм в длинноволновую область спектра. При рабочих параметрах лазера (температура лазерного диода $T_{2}=25^{\circ} \mathrm{C}$, температура активного световода $T_{1}=35^{\circ} \mathrm{C}$, мощность накачки 2,6 ВТ) были получены следующие выходные характеристики

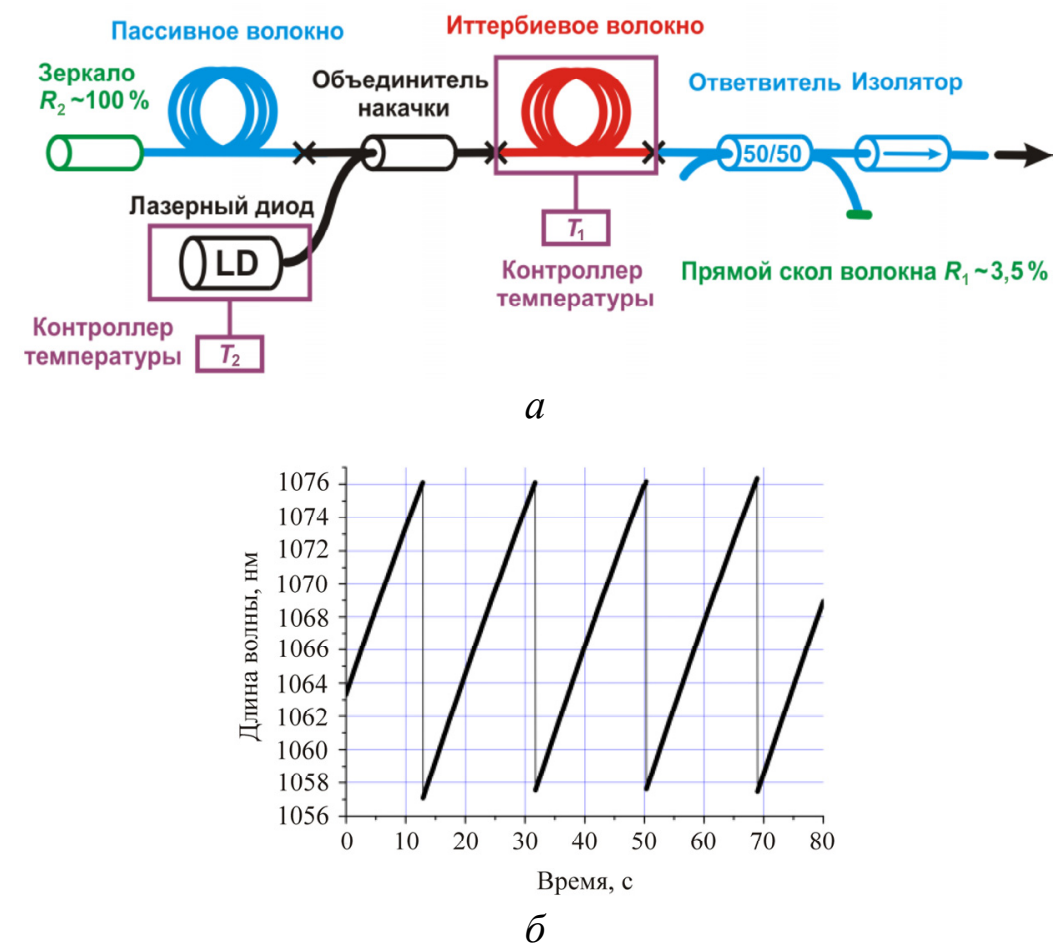

Рис. 1. Схема волоконного иттербиевого лазера с пассивным сканированием частоты $(a)$; характерная динамика длины волны при рабочих параметрах лазера (б) 
лазера: область сканирования 1057-1076 нм (см. рис. 1, б), скорость сканирования $~ 0,8 \mathrm{Hм} / \mathrm{c}$, время одного сканирования 20 с, выходная мощность $\sim 55$ мВт, частота следования одночастотных импульсов $\sim 45$ кГц, длительность импульсов 22,7 мкс. Таким образом, пиковая мощность составляет более 400 мВт.

\section{2. Устройство опроса волоконных ВБР-сенсоров}

Неопределенность длины волны генерации и импульсный характер излучения накладывает конкретные требования к созданию устройства опроса на основе реализованного источника. Первый факт требует постоянного измерения длины волны генерации лазера. Относительная длина волны генерации измерялась с помощью волоконного интерферометра Маха-Цендера, а привязка осуществлялась с помощью реперной ВБР с известной длиной волны отражения. Второй факт требует применения определенных алгоритмов обработки получаемых сигналов. Для этого из общего сигнала интенсивности выделялись только импульсы. Функциональная схема устройства опроса представлена на рис. 2. Оптический сигнал от источника с самосканированием частоты проходит через ответвитель 20/80, и $20 \%$ мощности подается в оптический канал, содержащий реперную ВБР и сенсорную линию; $80 \%$ мощности излучения также делится вторым ответвителем 50/50. Далее часть излучения проходит через волоконный интерферометр Маха-Цендера с областью свободной дисперсии 600 МГц и через спектрально-селективный фильтр 1045/1095 нм. Спектрально-селективный фильтр дополнительно вносит амплитудную модуляцию с целью определения момента скачка длины волны. Третья часть излучения проходит без каких-либо изменений и служит опорным сигналом. Сигнал, отраженный от сенсорной линии и прошедший через интерферометр, и опорный сигнал регистрируются тремя быстрыми InGaAs фотодетекторами (полоса 1 ГГц). Далее сигналы с фотодетекторов оцифровываются 10 МГц АЦП (E20-10, LCard) и поступают в режиме реального времени на персональный компьютер через интерфейс USB2. Для обработки сигналов применялся алгоритм, аналогичный использованному в работе [16]. Программа обработки, функционирующая в режиме реального времени, была реализована на языке LabView. На первом этапе обработки в сигнале опорного канала осуществлялся поиск положений импульсов. Далее выполнялось поимпульсное 


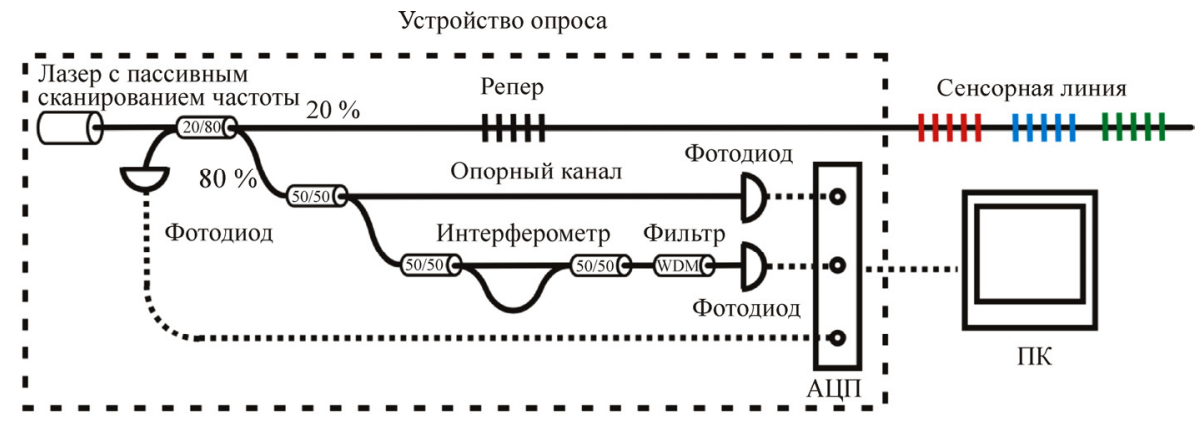

Рис. 2. Схема устройства опроса на базе волоконного лазера с самосканированием частоты

деление каналов с интерферометром и ВБР-сенсорами на опорный канал. Таким образом, после этой процедуры каждая точка в нормированных сигналах соответствует одной оптической частоте. С помощью интерферометра, фильтра и реперной ВБР (длина волны отражения 1066 нм) восстанавливалась временная динамика длины волн, аналогичная представленной на рис. 1, б. После этого восстанавливался спектр отражения сенсорной линии.

\section{3. Апробация устройства опроса}

Для апробации устройства опроса была реализована сенсорная линия из 6 ВБР (1060 нм, 1062 нм, 1064 нм, 1068 нм, 1072 нм, 1074 нм) на длины волн, попадающих в область сканирования лазера и не совпадающих с длиной волны реперной ВБР. Характерный спектр отражения сенсорной линии представлен на рис. 3, a. Для демонстрации воспроизведения данных на рисунке наложено 4 реализации. Также на рис. 3, б приведен измеренный спектр отражения реперной ВБР.

Для апробации устройства опроса один ВБР-датчик с длиной волны отражения вблизи 1074 нм был помещен в термостат, который позволяет нагревать волокно до $90{ }^{\circ} \mathrm{C}$. Нагрев приводил к смещению спектра длины волны отражения датчика в длинноволновую область спектра. Было проведено сравнение полученной температурной зависимости датчика с аналогичной зависимостью, полученной с помощью анализатора оптических спектров (AQ6370 Yakogawa) (рис. 4). Полученные результаты показывают, что измерения, сделанные с помощью устройства опроса и коммерческого спектроанализатора, полностью согласуются между собой. 


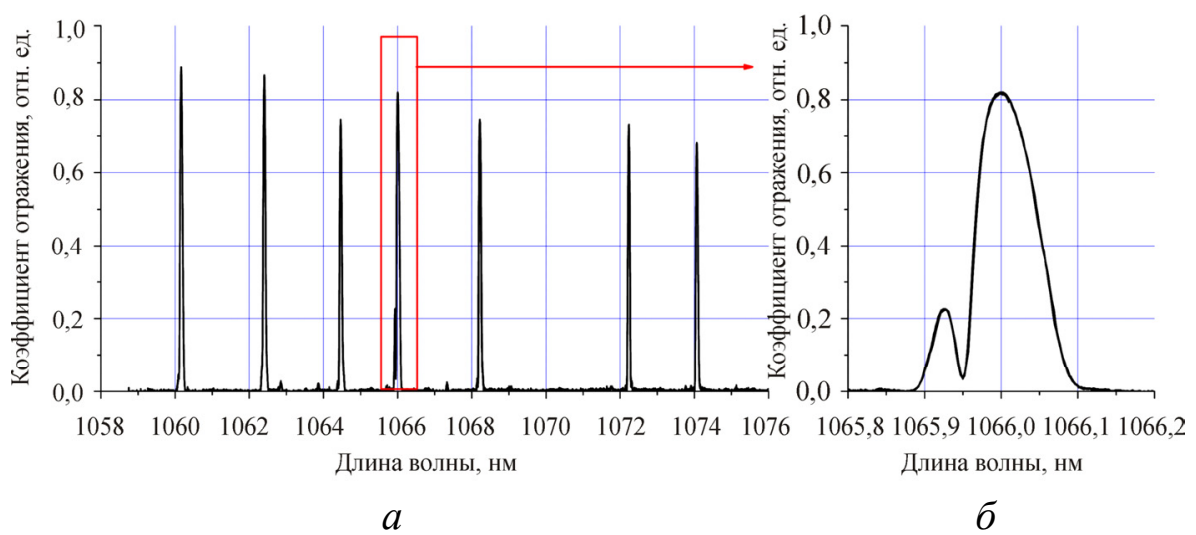

Рис. 3. Спектры отражения сенсорной линии ( $a$ ) и реперной ВБР (б), измеренные с помощью разработанного устройства опроса. На рисунке наложено 4 реализации

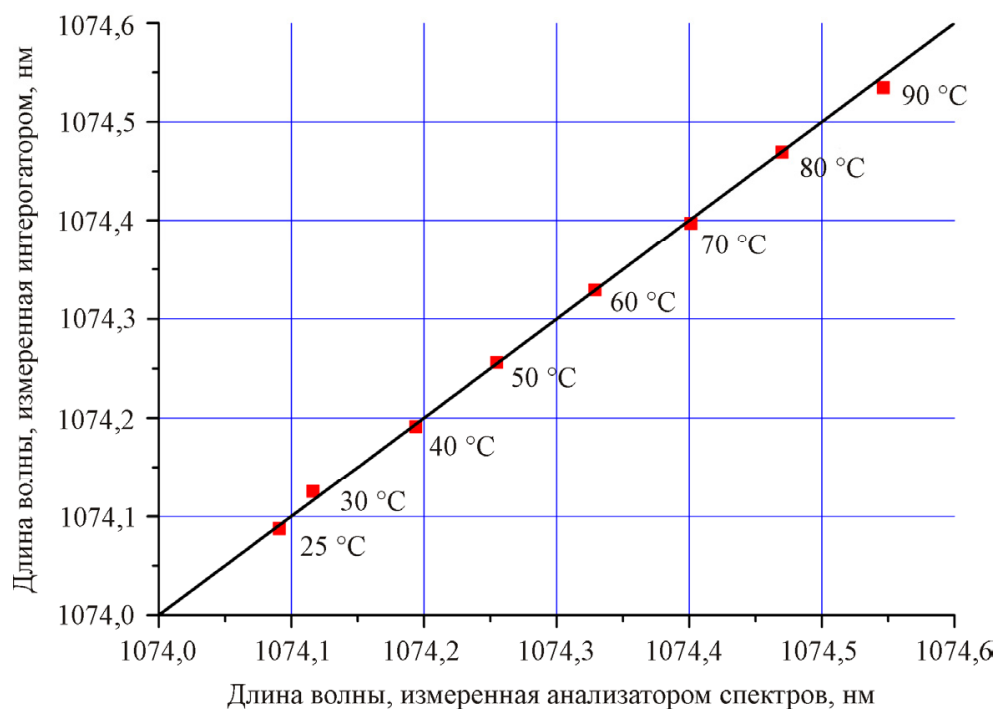

Рис. 4. Сравнение измерений длин волны отражения точечного датчика, произведенных с помощью разработанного устройства опроса и анализатора оптических спектров при разных температурах ВБР-датчика

Поскольку каждая точка в спектре отражения соответствует одной оптической частоте, устройство опроса позволяет прописывать спектры отражения ВБР-датчиков со спектральным разрешением порядка 5,5 МГц ( 20 фм), что определяется скачком частоты между импульсами. Стоит отметить, что спектральное разрешение коммерческих спектроанализаторов составляет 10 пм, это на три порядка боль- 
ше, чем в реализованном устройстве опроса. Точность определения длины волны максимума отражения решетки определяется как областью свободной дисперсии интерферометра ( 600 МГц), так и ширинами ВБР ( 30 ГГц). Несмотря на то что ширины спектров отражения репера и сенсоров достаточно велики, точность определения положения максимума была сравнима с областью свободной дисперсии интерферометра. Это было обеспечено применением алгоритма поиска максимума отражения с аппроксимацией квадратичным полиномом. Таким образом, точность определения спектра составила 600 МГц ( 2 пм). Это означает, что устройство позволяет определять температуру ВБР датчиков с точностью 0,3 град. Скорость опроса датчиков составила $\sim 0,05$ Гц, что определяется скоростью сканирования и диапазоном сканирования. Полученный диапазон сканирования 18 нм позволяет измерять до 9 датчиков в одноканальном режиме при спектральном расстоянии между датчиками 2 нм.

\section{Заключение}

Таким образом, в ходе исследования был разработан полностью волоконный одночастотный иттербиевый лазер с самосканированием частоты в области 1058-1076 нм и выходной мощностью более 50 мВт. При этом в лазере есть возможность смещения диапазона сканирования до 8 нм с помощью нагрева активного волокна. На основе лазера реализован прототип устройства опроса ВБР-датчиков. Устройство опроса апробировано на сенсорной линии, состоящей из 6 ВБР-датчиков. Точность определения длины волны отражения датчиков оценена как 2 пм, что сравнимо с имеющимися коммерческими аналогами. При этом спектральное разрешение реализованного устройства опроса на порядки превосходит аналоги. Относительно большая пиковая мощность излучения позволяет использовать устройство в многоканальном режиме, т.е. для одновременного опроса множества сенсорных линий. Стоит отметить, что некоторые характеристики предлагаемого устройства опроса уступают аналогам, работающим на основе перестраиваемых лазеров с использованием специальных перестроечных элементов. Например, в лазере с перестройкой частоты за счет модуляции тока накачки в резонаторе с большим значением дисперсии [7] была получена перестройка более 100 нм и скорость сканирования несколько миллионов нанометров в секунду. Стоит отметить, что для реальных ВБР- 
сенсорных систем, как правило, требуется диапазон сканирования более 40 нм со скоростью более 10 нм/с. Как показано в работе [17], диапазон сканирования может быть увеличен до более чем 20 нм с помощью тщательно подбора элементов лазера. Полученный диапазон сканирования 18 нм позволяет измерять до 9 датчиков в одноканальном режиме при спектральном расстоянии между датчиками 2 нм. Скорость же сканирования может быть увеличена с помощью повышения частоты повторения импульсов (до 200 кГц в [18]) и/или скачка частоты между импульсами (до 12 МГц в [12]). Таким образом, можно ожидать увеличения скорости сканирования до 10 нм/с, что близко к требуемым значениям. К недостаткам устройства опроса можно отнести неоптимальность спектральной области сканирования, так как значительная часть коммерчески доступных ВБР-датчиков работает в области прозрачности оптоволокна 1,5 мкм. К сожалению, на данный момент область самосканирования лазера с генерацией в области 1,5 мкм составляет менее 1 нм [14], что делает его малопригодным для практических приложений. Альтернативой могут служить лазеры на основе висмутовой активной среды. В работе [13] была продемонстрирована работа висмутового лазера с самосканированием частоты, с диапазоном сканирования более 10 нм в области 1,46 мкм, что достаточно близко к окну прозрачности оптоволокна. Дальнейшее смещение может быть достигнуто за счет подбора состава волокна и длины волны накачки.

\section{Список литературы}

1. Возможности, задачи и перспективы волокнно-оптических измерительных систем в современном приборостроении / В.Б. Гармаш, Ф.А. Егоров, Л.Н. Коломиец, А.П. Неугодников, В.И. Поспелов // Фотон-Экспресс. - 2005. - № 6. - С. 128-140.

2. Kashyap R. Fiber Bragg Gratings // Academic Press, 1999.

3. Сенсорная система на основе волоконно-оптических брэгговских решеток / С.А. Бабин, А.А. Власов, С.И. Каблуков, И.С. Шелемба // Вестник НГУ. Серия Физика. - 2007. - № 3. - С. 54-57.

4. Dawson J.W., Park N., Vahala K.J. Co-lasing in an electrically tunable erbium-doped fiber laser // Appl. Phys. Lett. - 1992. - № 60. P. 3090-3092. 
5. Dynamic sensor interrogation using wavelength-swept laser with a polygon-scanner-based wavelength filter / Y.S. Kwon, M.O. Ko, M.S. Jung, I.G. Park, N. Kim, S.-P. Han, H.-C. Ryu, K.H. Park, M.Y. Jeon // Sensors. 2013. - № 13. - P. 9669-9678.

6. Babin S.A., Kablukov S.I., Vlasov A.A. Tunable fiber Bragg gratings for application in tunable fiber lasers // Laser Physics. - 2007. - № 17. P. 1323-1326

7. Yamashita S., Takubo Y. Wide and fast wavelength-swept fiber lasers based on dispersion tuning and their application to optical coherence tomography // Photonic Sensors. - 2013. - № 3. - p. 320-331.

8. Characterization of FBG sensor interrogation based on a FDML wavelength swept laser / E.J. Jung, C.-S. Kim, M.Y. Jeong, M.K. Kim, M.Y. Jeon, W. Jung, Z. Chen // Opt. Express. - 2008. - № 16. - P. $16552-$ 16560.

9. Hughes T.P., Young K.M. Mode sequences in ruby laser emission // Nature. - 1962. - № 196. - P. 332-334.

10. Kir'yanov A.V., Il'ichev N.N. Self-induced laser line sweeping in an ytterbium fiber laser with nonresonant Fabry-Perot cavity // Laser Phys. Lett. - 2011. - № 8. - P. 305-312.

11. Broad-range self-sweeping of a narrow-line self-pulsing Yb-doped fiber laser / I.A. Lobach, S.A. Babin, S.I. Kablukov, E.V. Podivilov // Opt. Express. - 2011. - № 19. - P. 17632-17640.

12. Self-scanned single-frequency operation of a fiber laser driven by a self-induced phase grating / I.A. Lobach, S.I. Kablukov, E.V. Podivilov, S.A. Babin // Laser Phys. Lett. - 2014. - № 11. - p. 045103.

13. Single-frequency Bismuth-doped fiber laser with quasicontinuous self-sweeping / I.A. Lobach, S.I. Kablukov, M.A. Melkumov, V.F. Khopin, S.A. Babin, E.M. Dianov // Opt. Express. - 2015. - № 23. P. 24833-24842.

14. Self-induced laser line sweeping in tunable erbium-doped fiber laser / P. Honzátko, P. Vojtíšek, P. Navrátil, P. Peterka // Proc. 5th EPSQEOD Europhoton conf., WeP. 28. - Stockholm, Sweden, 2012.

15. Tm-Ho co-doped all-fiber brand-range self-sweeping laser around 1,9 um / X. Wang, P. Zhou, X. Wang, H. Xiao, L. Si // Opt. Express. 2013. - № 21. - P. 16290-16295. 
16. Lobach I.A., Kablukov S.I. Application of a self-sweeping Yb-doped fiber laser for high-resolution characterization of phase-shifted FBGs // J. Lightwave Technol. - 2013. - № 31. - P. 2982-2987.

17. Lobach I.A., Tkachenko A.Yu., Kablukov S.I. Optimization and control of sweeping range in Yb-doped self-sweeping fiber laser // Laser Phys. Lett. - 2016. (Принято к печати.)

18. Fourier synthesis with single-mode pulses from a multimode laser / I.A. Lobach, S.I. Kablukov, E.V. Podivilov, A.A. Fotiadi, S.A. Babin // Opt. Lett. - 2015. - № 40. - p. 3671-3674.

\section{References}

1. Garmash V.B., Egorov F.A., Kolomiets L.N., Neugodnikov A.P., Pospelov V.I. Vozmozhnosti, zadachi i perspektivy voloknno-opticheskikh izmeritel'nykh sistem $\mathrm{v}$ sovremennom priborostroenii [Opportunities, challenges and prospects of optical fiber measuring systems in modern instrument engineering]. Foton-Ekspress, 2005, no. 6, pp. 128-140.

2. Kashyap R. Fiber Bragg Gratings. San Diego: Academic Press, 1999. $478 \mathrm{p}$.

3. Babin C.A., Vlasov A.A., Kablukov S.I., Shelemba I.S. Sensornaia sistema na osnove volokonno-opticheskikh breggovskikh reshetok [Sensor system based on fiber optic Bragg gratings]. Vestnik Novosibirskogo gosudarstvennogo universiteta. Seriia “Fizika", 2007, no. 3, pp. 54-57.

4. Dawson J.W., Park N., Vahala K.J. Co-lasing in an electrically tunable erbium-doped fiber laser. Appl. Phys. Lett., 1992, no. 60, pp. 30903092 .

5. Kwon Y.S., Ko M.O., Jung M.S., Park I.G., Kim N., Han S.-P., Ryu H.-C., Park K.H., Jeon M.Y. Dynamic sensor interrogation using wavelength-swept laser with a polygon-scanner-based wavelength filter. Sensors, 2013, no, 13, pp. 9669-9678.

6. Babin S.A., Kablukov S.I., Vlasov A.A. Tunable fiber Bragg gratings for application in tunable fiber lasers. Laser Physics, 2007, no. 17, pp. 1323-1326.

7. Yamashita S., Takubo Y. Wide and fast wavelength-swept fiber lasers based on dispersion tuning and their application to optical coherence tomography. Photonic Sensors, 2013, no. 3, pp. 320-331.

8. Jung E.J., Kim C.-S., Jeong M.Y., Kim M.K., Jeon M.Y., Jung W., Chen Z. Characterization of FBG sensor interrogation based on 
a FDML wavelength swept laser. Opt. Express, 2008, no. 16, pp. 1655216560 .

9. Hughes T.P., Young K.M. Mode sequences in ruby laser emission. Nature, 1962, no. 196, pp. 332-334.

10. Kir'yanov A.V., Il'ichev N.N. Self-induced laser line sweeping in an ytterbium fiber laser with nonresonant Fabry-Perot cavity. Laser Phys. Lett., 2011, no. 8, pp. 305-312.

11. Lobach I.A., Babin S.A., Kablukov S.I., Podivilov E.V. Broadrange self-sweeping of a narrow-line self-pulsing Yb-doped fiber laser. Opt. Express, 2011, no. 19, pp. 17632-17640.

12. Lobach I.A., Kablukov S.I., Podivilov E.V., Babin S.A. Selfscanned single-frequency operation of a fiber laser driven by a self-induced phase grating. Laser Phys. Lett., 2014, no. 11, pp. 045103.

13. Lobach I.A., Kablukov S.I., Melkumov M.A., Khopin V.F., Babin S.A., Dianov E.M. Single-frequency Bismuth-doped fiber laser with quasicontinuous self-sweeping. Opt. Express, 2015, no. 23, pp. 24833-24842.

14. Honzátko P., Vojtíšek P., Navrátil P., Peterka P. Self-induced laser line sweeping in tunable erbium-doped fiber laser. Proc. 5th EPS-QEOD Europhoton conf. Stockholm, 2012. WeP.28.

15. Wang X., Zhou P., Wang X., Xiao H., Si L. Tm-Ho co-doped allfiber brand-range self-sweeping laser around 1.9 um. Opt. Express, 2013, no. 21, pp. 16290-16295.

16. Lobach I.A., Kablukov S.I. Application of a self-sweeping Ybdoped fiber laser for high-resolution characterization of phase-shifted FBGs. J. Lightwave Technol., 2013, no. 31, pp. 2982-2987.

17. Lobach I.A., Tkachenko A.Yu., Kablukov S.I. Optimization and control of sweeping range in Yb-doped self-sweeping fiber laser. Laser Phys. Lett., 2016. (Accepted for publication.)

18. Lobach I.A., Kablukov S.I., Podivilov E.V., Fotiadi A.A., Babin S.A. Fourier synthesis with single-mode pulses from a multimode laser. Opt. Lett., 2015, no. 40, pp. 3671-3674.

Получено 13.03.2016 